\title{
A New Automated Method and Sample Data Flow for Analysis of Volatile Nitrosamines in Human Urine*
}

\author{
James A. Hodgson"1,2, Tiffany H. Seyler1\#, Ernest McGahee1, Stephen Arnstein', \\ Lanqing Wang 1 \\ ${ }^{1}$ Tobacco and Volatiles Branch, Division of Laboratory Sciences, National Center for Environmental Health, \\ Centers for Disease Control and Prevention, Atlanta, USA \\ ${ }^{2}$ Oak Ridge Institute for Science and Education (ORISE), Oak Ridge, USA \\ Email: "tvh2@cdc.gov
}

Received 30 December 2015; accepted 30 January 2016; published 2 February 2016

Copyright (C) 2016 by authors and Scientific Research Publishing Inc.

This work is licensed under the Creative Commons Attribution International License (CC BY).

http://creativecommons.org/licenses/by/4.0/

(c) (i) Open Access

\section{Abstract}

Volatile nitrosamines (VNAs) are a group of compounds classified as probable (group 2A) and possible (group 2B) carcinogens in humans. Along with certain foods and contaminated drinking water, VNAs are detected at high levels in tobacco products and in both mainstream and sidestream smoke. Our laboratory monitors six urinary VNAs- $\mathrm{N}$-nitrosodimethylamine (NDMA), Nnitrosomethylethylamine (NMEA), N-nitrosodiethylamine (NDEA), N-nitrosopiperidine (NPIP), N-nitrosopyrrolidine (NPYR), and N-nitrosomorpholine (NMOR)-using isotope dilution GC-MS/ MS (QQQ) for large population studies such as the National Health and Nutrition Examination Survey (NHANES). In this paper, we report for the first time a new automated sample preparation method to more efficiently quantitate these VNAs. Automation is done using Hamilton STAR ${ }^{\mathrm{TM}}$ and Caliper Staccato ${ }^{\mathrm{TM}}$ workstations. This new automated method reduces sample preparation time from 4 hours to 2.5 hours while maintaining precision (inter-run CV $<10 \%)$ and accuracy $(85 \%$ 111\%). More importantly this method increases sample throughput while maintaining a low limit of detection $(<10 \mathrm{pg} / \mathrm{mL})$ for all analytes. A streamlined sample data flow was created in parallel to the automated method, in which samples can be tracked from receiving to final LIMs output with minimal human intervention, further minimizing human error in the sample preparation process. This new automated method and the sample data flow are currently applied in biomonitoring of VNAs in the US non-institutionalized population NHANES 2013-2014 cycle.

\footnotetext{
*The findings and conclusions in this report are those of the authors and do not necessarily represent the official position of the Centers for Disease Control and Prevention. Use of trade names and commercial sources is for identification only and does not constitute endorsement by the U.S. Department of Health and Human Services, or the U.S. Centers for Disease Control and Prevention.

"Corresponding author.
}

How to cite this paper: Hodgson, J.A., Seyler, T.H., McGahee, E., Arnstein, S. and Wang, L.Q. (2016) A New Automated Method and Sample Data Flow for Analysis of Volatile Nitrosamines in Human Urine. American Journal of Analytical Chemistry, 7, 165-178. http://dx.doi.org/10.4236/ajac.2016.72014 


\section{Keywords}

\section{Volatile Nitrosamines, Automation, Sample Data Flow, Gas Chromatography, Tandem Mass Spectrometry}

\section{Introduction}

Volatile nitrosamines (VNAs) are a class of nitrosated secondary and tertiary amines (Figure 1). VNAs are known carcinogens and teratogens in animals and are classified as group 2A and 2B carcinogens in humans [1][9]. They have been shown to induce tumors via cytochrome-activated DNA alkylation in several organs, including liver, lungs, kidney, bladder, pancreas, and esophagus [1] [5]-[7] [9] [10]. VNAs may lead to lipid peroxidation and oxidative stress, as well as chronic diseases such as diabetes and Alzheimer's disease [11]-[18].

The formation of volatile nitrosamines occurs through the nitrosation of secondary and tertiary amines via interaction with nitrite, which itself is a product of nitrate reduction [3]-[5] [7] [8] [19] [20]. For this reason, VNAs can be formed from many items containing nitrates and nitrites, such as cured meats, fish products, cosmetics, certain types of beers, and tobacco products (as part of the curing process and during product assembly), as well as in both mainstream and sidestream tobacco smoke [2] [4] [7] [21]-[23]. Another significant source of VNA exposure can be drinking water: VNAs, particularly NDMA, can form as byproducts during disinfection via chlorination and chloramination [2] [9] [24]-[28].

Many different methods are reported for VNA measurements. For sample preparation, dichloromethane is the solvent of choice for extracting VNAs from the sample matrix (e.g. water, urine, serum), whether in a direct liquidliquid extraction or using a solid phase intermediary [9] [24]. As for instrumentation, gas chromatography is the most common separation technique due to the eponymous volatility of VNAs, though some labs have developed various liquid chromatography methods [2] [5] [6] [22] [23] [27]. Detection methods range from single- and triple-quadrupole mass spectrometry to thermal energy analysis and nitrogen chemiluminescence detection, though mass spectrometry is the most commonly used [1] [8] [19] [20] [25] [26] [28].

Automation is necessary for higher sample throughput in large population studies such as NHANES, whose sample size is approximately 10,000 per two-year cycle. In this study, we present an automated method utilizing both Caliper Staccato and Hamilton Star workstations. The throughput is increased by automation equipment, and the sensitivity is increased due in part to an upgrade of the QQQ from an Agilent 7000B to a 7000C. We created a streamlined sample data flow in parallel to the automated method, in which samples can be tracked from receiving to final LIMs output with minimal human intervention, further minimizing human error in the sample preparation process.

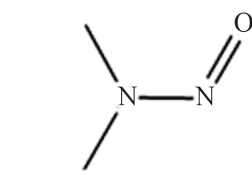

$\mathrm{N}$-nitrosodimethylamine NDMA

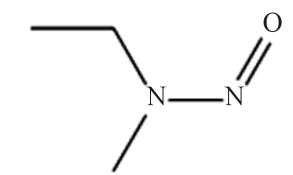

$\mathrm{N}$-nitrosomethylethylamine NMEA

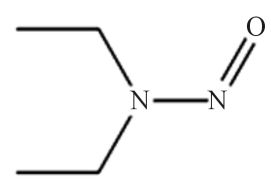

N-nitrosodiethylamine NDEA

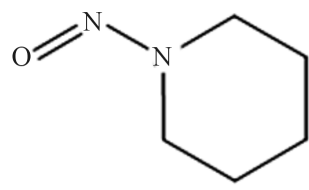

N-nitrosopiperidine NPIP

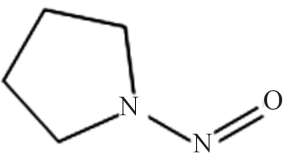

$\mathrm{N}$-nitrosopyrrolidine NPYR

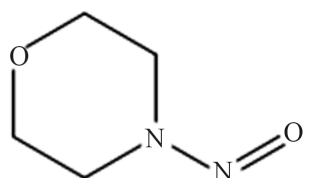

$\mathrm{N}$-nitrosomorpholine NMOR

Figure 1. Structures of volatile nitrosamines. 


\section{Methods and Materials}

\subsection{Materials}

Native standards were purchased from Supelco (Sigma-Aldrich, St. Louis, MO) as a $2 \mathrm{mg} / \mathrm{mL}$ mixture in dichloromethane (DCM). Deuterium-labeled internal standards NDMA-D6, NDEA-D10, NPYR-D8, and NMORD8 were purchased individually in DCM from Cambridge Isotope Laboratories (Tewksbury, MA); NMEA-D3 and NPIP-D10 were purchased individually as pure oils from Toronto Research Chemicals (Toronto, Canada). Methanol (MeOH), DCM, and acetonitrile (ACN) were HPLC grade, purchased from Honeywell Burdick \& Jackson (Muskegon, MI). Sample plates were Axygen 48-well plates with a $5 \mathrm{~mL}$ well capacity. GC vials were Wheaton $11 \mathrm{~mm}$ amber crimp vials with a $300 \mu \mathrm{L}$ insert; crimp caps were SUN-SRi $11 \mathrm{~mm}$ aluminum crimp caps with rubber septum. All GC-QQQ parts were purchased from Agilent Technologies (Santa Clara, CA).

\subsection{Hamilton Microlab Star Liquid Handling Workstation}

All sample and internal standard aliquoting was performed on the Hamilton Microlab Star liquid handling workstation. The pipetting array consisted of 8 pipetting heads and used $1000 \mu \mathrm{L}$ and $50 \mu \mathrm{L}$ compressed O-ring expansion (CO-RE) tips. All samples were aspirated using capacitance liquid level detection (cLLD) and dispensed using the jet empty setting. Samples were mixed (consisting of an aspirate of $500 \mu \mathrm{L}$ and dispense into the same container) 3 times prior to being transferred to the sample plate to ensure sample uniformity.

\subsection{Caliper Staccato Specimen Handling Workstation}

Sample preparation was performed on the Caliper Staccato specimen handling workstation, including an integrated Perkin Elmer SciClone G3 Automated Liquid Handling Workstation (Figure 2). This system was custom designed with all specimen preparation needs in mind. The Staccato station includes the SciClone G3 Workstation, a Biotage TurboVap 96 Automated Evaporation System, a Hettich GmbH \& Co. KG Rotanta 460 Robotic Centrifuge, two HyperStak39 consumable loaders, a Thermo Scientific ALPS 3000 sealer, and a Mitsubishi S Series Melfa RV-6SDL Industrial Robot.

\subsection{Automated Sample Preparation}

Urine samples were transferred from cryovials in $2 \mathrm{~mL}$ aliquots, along with $500 \mathrm{pg}$ of internal standard (in methanol solution, $10 \mathrm{pg} / \mu \mathrm{L}$ ), into a 48-well plate using a Hamilton Star liquid handling system. The delivered volume of $50 \mu \mathrm{L}$ was verified gravimetrically with \% error of less than $1 \%$. The sample plate was transferred to a SciClone liquid handler on the Caliper Staccato specimen handling workstation, where $2.5 \mathrm{~mL}$ of DCM were added to each well. All samples underwent pipette mixing for approximately 40 minutes using the 96-head main array and $200 \mu \mathrm{L}$ pipette tips. The plate was sealed using a Thermo Scientific ALPS 3000 sealer and centrifuged for 5 minutes $\left(1500 \mathrm{rpm}, 25^{\circ} \mathrm{C}\right)$ in a Hettich Rotanta 460 Robotic centrifuge. The sample plate was then moved back onto the SciClone deck, where the seal was pierced, and $1.5 \mathrm{~mL}$ of the DCM layer in each well were transferred to a new 48-well plate. Samples were evaporated to approximately $300 \mu \mathrm{L}$ in a Biotage 96-array TurboVap, and the plate was sealed and transferred off the Caliper Staccato system. Each sample was then manually transferred via pipette to a $1 \mathrm{~mL}$ amber GC vial with a $300 \mu \mathrm{L}$ insert and further evaporated to approximately $100 \mu \mathrm{L}$ in a ThermoFisher Savant SPD2010 Speedvac Concentrator. To each sample $50 \mu \mathrm{L}$ of ACN were added, then the remaining DCM was evaporated in the Savant. Sample vials were sealed using aluminum crimp caps and transferred to GC-MS/MS (QQQ) for analysis (Figure S1).

\subsection{GC-MS/MS (QQQ) Analysis}

All analyses were performed on an Agilent 7890-7000C GC-MS/MS (QQQ). The 7890 GC was equipped with a multimode inlet (MMI) and a single taper helix liner. The injection volume was $5 \mu \mathrm{L}$. The initial injection temperature was $5^{\circ} \mathrm{C}$, which was held for 0.85 minutes after injection and then heated at $600^{\circ} \mathrm{C} / \mathrm{min}$ to $300^{\circ} \mathrm{C}$. A programmed temperature vaporation (PTV) solvent vent mode was used, venting at $200 \mathrm{~mL} / \mathrm{min}$ flow rate and 5 psi pressure for 0.7 minutes after injection. A two-column setup connected by a backflush union was used for the GC, with helium as the carrier gas. The first column was an Agilent DB-WAXetr ( $30 \mathrm{~m} \times 0.25 \mathrm{~mm} \times 0.5 \mu \mathrm{m})$ with a constant flow rate of $3 \mathrm{~mL} / \mathrm{min}$ for the first 1.3 minutes, followed by a $1.2 \mathrm{~mL} / \mathrm{min}$ flow rate for the 

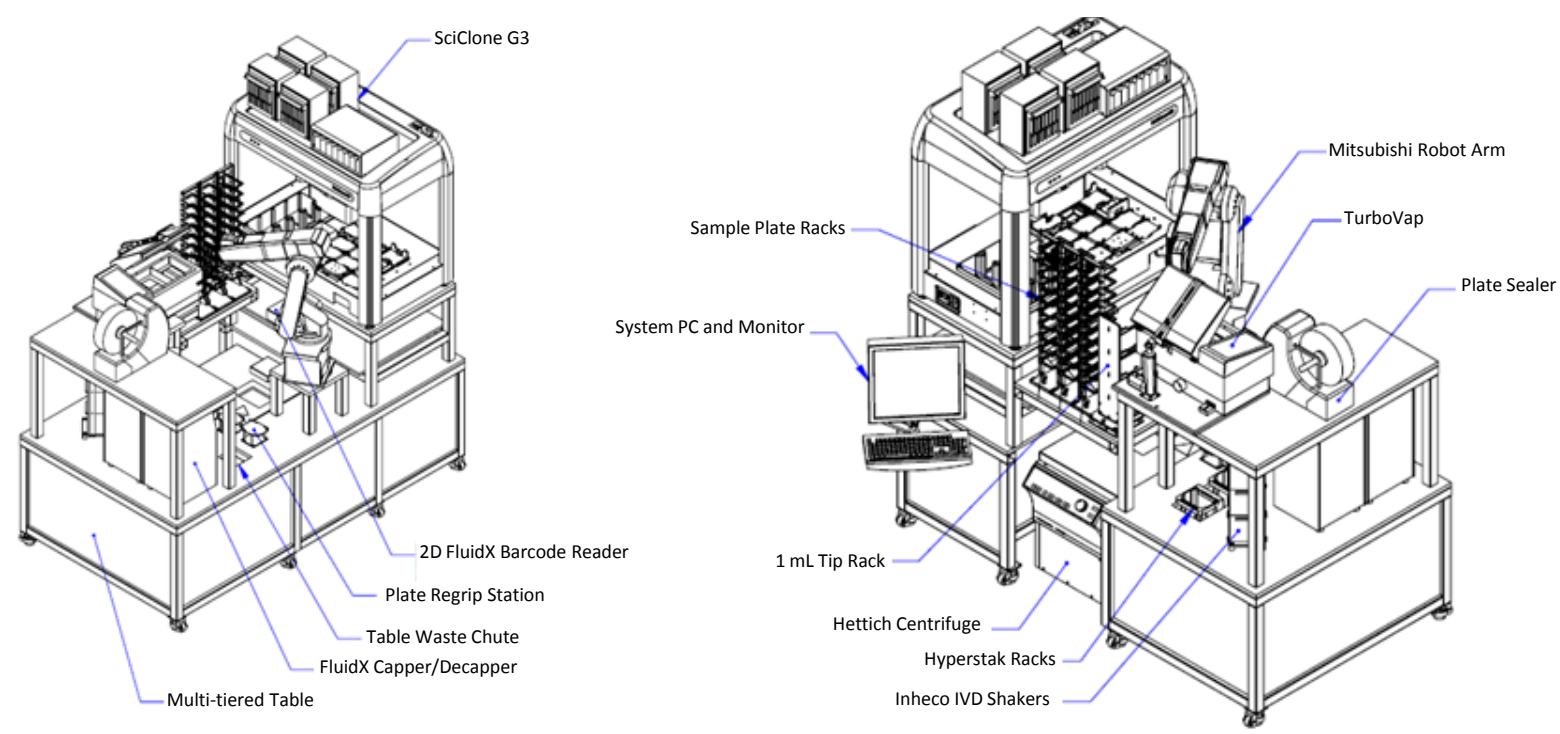

Figure 2. Schematic for Caliper Staccato workstation.

remainder of the run. The second column was deactivated fused silica $(1 \mathrm{~m} \times 0.15 \mu \mathrm{m})$ with a constant pressure of 1 psi. The GC oven was initially set to $35^{\circ} \mathrm{C}$ for 1 minute after injection then heated to $245^{\circ} \mathrm{C}$ at $20^{\circ} \mathrm{C} / \mathrm{min}$. A backflush was performed for 5 minutes post-run, with a $-1.9795 \mathrm{~mL} / \mathrm{min}$ constant flow for the first column, a 25 psi constant pressure for the second column, and an oven temperature of $250^{\circ} \mathrm{C}$. The transfer line temperature and MS source were both set to $250^{\circ} \mathrm{C}$. The $7000 \mathrm{C}$ MS source mode was positive chemical ionization (CI) with ammonia (blue grade) as the CI gas; ultra-high purity nitrogen was used as the collision gas.

\subsection{Sample Data Flow}

A mostly automated system for tracking sample data was created in parallel with the automated sample preparation method (Figure 3). Samples are received and logged into the LIMs reporting system before they are queued for preparation and analysis. Samples to be prepared are scanned by the Hamilton Star, which upon completion generates an output file mapping scanned samples to positions in the 48-well plate. This Hamilton output file is run through an Excel macro which modifies the format so that it can be imported into MassHunter as a sequence file. Once the raw data are acquired from the GC-QQQ, they can either be analyzed in MassHunter Quantitative software or uploaded to Indigo Biosystems ASCENT platform for automatic integration. Regardless of which quantitation software is used, a formatted output file containing the final calculated data is generated and can be directly uploaded to the LIMs system. Using a custom LIMs system, unknown samples are evaluated individually according to a list of QA rules, including retention times of internal standard and main ion transition peaks, confirmation ion ratio, internal standard peak area, and blank limit. Batch QCs are evaluated according to modified Westgard QC rules [29]. Final results are then exported to a final reporting system such as NHANES.

\section{Results}

\subsection{Blanks}

A true blank has proven difficult to produce, as VNAs—-particularly NDMA-are detected in all water sources tested thus far, including Fluka Analytical TraceSELECT ultratrace water (Sigma-Aldrich), a commercial source of VOC-free water, and in-house filtered "VOC-free" water. NDMA contamination has also been detected when DCM (the extraction solvent) comes into contact with any plastic consumable used during the sample preparation process, such as the 48-well plates and pipette tips. As a result, DCM is processed as an unknown sample and used as the system blank and for blank subtraction. The concentration of NDMA contamination seen in DCM blank samples is approximately 1 - 2 times the LOD reported here. The first and last well in the plate contain DCM, spiked with 500 pg ISTD, and carried all the way through the sample preparation process. The average calculated concentration derived from the analytical results of these two DCM blanks is 

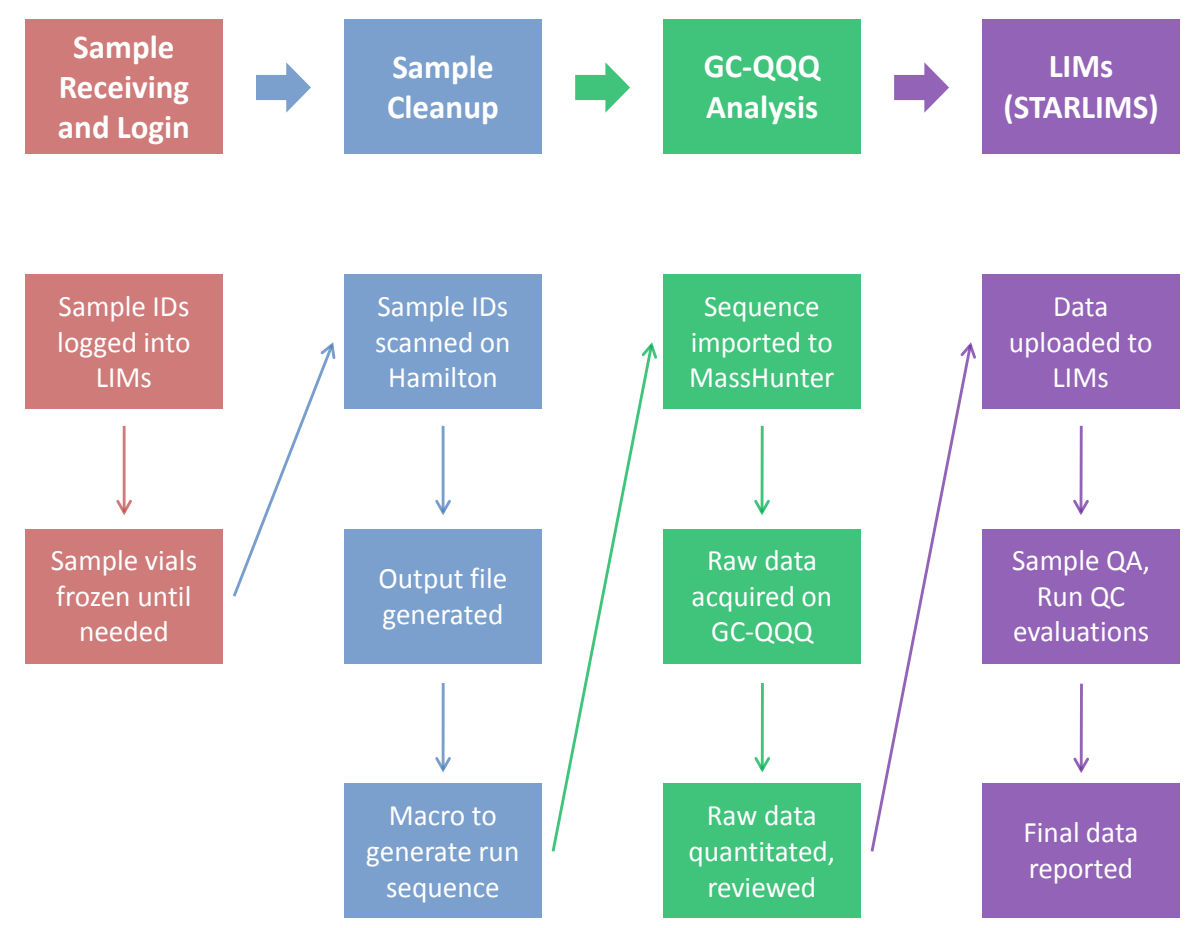

Figure 3. Sample data flow chart.

then subtracted from all analytical results for the remainder of that batch (i.e. all other samples prepared in the same 48-well plate). The blank subtraction is built into the process of data loading to LIMs. Blank characterization was established over 60 separate runs over a one year period. Runs are rejected if any DCM blank result exceeds the established blank limits for each analyte.

\subsection{Carryover}

Acetonitrile blanks were run immediately after high $(200 \mathrm{pg} / \mathrm{mL})$ QC samples. These blanks were then compared with blanks run without any immediately preceding sample. Calculated concentrations of the two sets of blanks were within $5 \%$ both of one another and of the blank characterization values, indicating no carryover. Some small carryover was observed for NDMA, NMEA, and NDEA after the injections of the highest standard, $400 \mathrm{ng} / \mathrm{mL}$. After 3 ACN solvent blank injections, there is no carryover observed for these three analytes. Thus, ACN solvent blank is injected three times after the highest standard in every analytical batch. As a precaution, a QA rule is built into the LIMs system to flag a sample immediately following a high concentration sample ( $>200$ $\mathrm{pg} / \mathrm{mL}$ ). The flagged samples are reinjected to ensure no carryover occurred. Carryover is determined by established repeatability criteria: $20 \%$ for lower concentration $(<50 \mathrm{pg} / \mathrm{mL})$ and $10 \%$ for higher concentration $(\geq 50$ $\mathrm{pg} / \mathrm{mL}$ ). If a reinjected sample fails repeatability rules, it will be repeated. An ACN blank is also injected at the beginning of each analytical batch to ensure no system contamination exists prior to sample analysis.

\subsection{Limits of Detection (LOD)}

The limit of detection for each analyte was obtained from 60 independent runs (limited to only 1 run per day) using DCM blanks (carried through sample preparation process, as mentioned above), as well as prepared samples from 4 pools: $0 \mathrm{pg} / \mathrm{mL}, 2.5 \mathrm{pg} / \mathrm{mL}, 5.0 \mathrm{pg} / \mathrm{mL}$, and $7.5 \mathrm{pg} / \mathrm{mL}$. Because NDMA, NMEA, NDEA, NPIP, and NMOR have positive blank detections, these LODs were calculated using the $3 \mathrm{~S}_{0}$ method, where $\mathrm{S}_{0}$ is the extrapolated standard deviation at zero concentration. For NPYR, the LOD was determined according to the guideline for determination of limits of detection by the Clinical and Laboratory Standard Institute using the 4 QC pools [29]. The LODs for all analytes are below $10 \mathrm{pg} / \mathrm{mL}$, with three of the analytes (NMEA, NDEA, and NPIP) at or below $5 \mathrm{pg} / \mathrm{mL}$ (Table 1 ). 
Table 1. Limits of detection of all analytes (LODs were obtained from 60 independent runs, limited to one run per day).

\begin{tabular}{ll}
\hline & Limit of Detection $(\mathrm{pg} / \mathrm{mL})$ \\
NDMA & $6.54^{*}$ \\
NMEA & $3.64^{*}$ \\
NDEA & $5.02^{*}$ \\
NPIP & $5.08^{*}$ \\
NPYR & $8.15^{* *}$ \\
NMOR & $7.84^{*}$ \\
\hline
\end{tabular}

"Determined by $3 \mathrm{~S}_{0}$, where $\mathrm{S}_{0}$ is the standard deviation of blank characterization; ${ }^{* *}$ Calculated according to CLSI, using 4 pools $(0 \mathrm{pg} / \mathrm{mL}, 2.5 \mathrm{pg} / \mathrm{mL}$, $5.0 \mathrm{pg} / \mathrm{mL}, 7.5 \mathrm{pg} / \mathrm{mL}$ ).

\subsection{Precision}

To determine intra-run and inter-run precision, in-house anonymous non-smoker urine (collected with CDC Institutional Review Board (IRB) approval) was spiked to make two QC pools at $50 \mathrm{pg} / \mathrm{mL}$ and $200 \mathrm{pg} / \mathrm{mL}$. Six samples of each QC were run for five consecutive days. For the $50 \mathrm{pg} / \mathrm{mL} \mathrm{QC}$, only one analyte in one run has an intra-run CV greater than 10\%; the rest of the pool is below $7 \%$ CV. For the $200 \mathrm{pg} / \mathrm{mL}$ QC, all analytes are at or below 5\% intra-run CV. For both sets, all inter-run CVs are 5-10\% (Table 2).

\subsection{Accuracy in Solution}

To determine accuracy of the calibration curve, individual stocks of native NDMA, NDEA, NPYR, and NMOR were obtained from a different vendor, Cambridge Isotope Laboratories. Because no manufacturer readily carries individual stocks of native NMEA or NPIP, a different lot of the Supelco native VNA mixture was obtained. Three levels of calibrator (at $0.5 \%, 25 \%$, and $50 \%$ of the highest calibrator) were made and run in triplicate for each individual stock, as well as for the new mixture. For all three levels, the accuracy is greater than $94 \%$ for all analytes, both individually and in the mixture (Table 3). This test is repeated every time a new standard curve is prepared for analysis.

\subsection{Accuracy in Matrix}

To determine accuracy in matrix, freshly collected in-house anonymous non-smoker urine (collected with CDC Institutional Review Board (IRB) approval) was spiked at 3 different levels each day: $100 \mathrm{pg} / \mathrm{mL}, 200 \mathrm{pg} / \mathrm{mL}$, and $300 \mathrm{pg} / \mathrm{mL}$. These pools were prepared and run in triplicate for three consecutive days. For the $100 \mathrm{pg} / \mathrm{mL}$ spiked samples, the calculated accuracy for all analytes is $85 \%-111 \%$; for $200 \mathrm{pg} / \mathrm{mL}$ and $300 \mathrm{pg} / \mathrm{mL}$, the accuracy for all analytes is $92 \%-106 \%$ (Table 4).

\section{Discussion}

Automation of sample preparation processes is a crucial part of bio-monitoring large population studies like NHANES. Analyzing more than 10,000 samples per two-year cycle requires a much higher throughput than an analyst could perform manually. Our new automated method for preparing samples enables the analyst to achieve the necessary sample throughput while still maintaining high accuracy and precision. With detection limits for all analytes below $10 \mathrm{pg} / \mathrm{mL}$, and three of six at or below $5 \mathrm{pg} / \mathrm{mL}$, these LODs are comparable to the ones reported in Seyler, et al. [21]. Also, the staff time saved because of the automated steps can be re-allocated to instrument operation and data analysis, further increasing throughput.

The automated sample data flow further minimizes human error in sample handling. Sample IDs are first tracked during initial sample aliquoting on the Hamilton Star, where an output file automatically maps samples to well locations on the 48-well plate. The Hamilton output file is then converted to an imported GC-QQQ sequence file via an Excel macro. All relevant sample information, such as the sample ID, sample volume, and any dilution factors, is saved with each raw data file as the samples are analyzed on the GC-QQQ. The current 
Table 2. Precision.

\begin{tabular}{|c|c|c|c|c|c|c|}
\hline \multicolumn{7}{|l|}{ Pool $1-50$ pg/mL } \\
\hline & NDMA & NMEA & NDEA & NPIP & NPYR & NMOR \\
\hline Intra-run $(n=6)$ & 4.26 & 4.87 & 4.63 & 1.90 & 4.65 & 4.69 \\
\hline \multirow[t]{4}{*}{ CV (\%) } & 5.26 & 2.03 & 3.60 & 4.51 & 6.43 & 2.43 \\
\hline & 3.77 & 2.42 & 2.68 & 1.05 & 6.54 & 2.18 \\
\hline & 2.03 & 2.68 & 1.93 & 2.19 & 3.19 & 3.16 \\
\hline & 11.1 & 3.02 & 5.16 & 5.04 & 5.44 & 2.54 \\
\hline Inter-run $(\mathrm{n}=5)$ & 7.94 & 6.14 & 5.17 & 5.36 & 9.34 & 5.16 \\
\hline \multicolumn{7}{|l|}{ Pool $2-200$ pg/mL } \\
\hline & NDMA & NMEA & NDEA & NPIP & NPYR & NMOR \\
\hline Intra-run $(n=6)$ & 3.40 & 2.24 & 2.68 & 1.97 & 2.68 & 5.07 \\
\hline \multirow[t]{4}{*}{ CV (\%) } & 1.58 & 1.26 & 1.69 & 2.19 & 2.08 & 1.24 \\
\hline & 1.69 & 2.91 & 2.33 & 2.67 & 2.75 & 2.49 \\
\hline & 1.49 & 2.16 & 2.76 & 2.98 & 2.29 & 2.20 \\
\hline & 3.97 & 3.27 & 3.55 & 3.23 & 4.16 & 3.32 \\
\hline Inter-run $(\mathrm{n}=5)$ & 7.44 & 7.09 & 6.78 & 6.95 & 5.70 & 6.12 \\
\hline
\end{tabular}

Table 3. Accuracy in solution.

\begin{tabular}{ccccccc}
\hline $\begin{array}{c}\text { Accuracy }(\%) \\
\mathrm{n}=3\end{array}$ & NDMA & NMEA & NDEA & NPIP & NPYR & NMOR \\
Mixed—2 ng/mL & 99.9 & 95.5 & 95.4 & 94.8 & 96.2 & 94.2 \\
Mixed—100 ng/mL & 100 & 101 & 100 & 101 & 98.7 & 102 \\
Mixed—200 ng/mL & 101 & 103 & 101 & 100 & 98.7 & 102 \\
Individual-2 ng/mL & 95.7 & & 94.7 & & 96.7 & 98.0 \\
Individual-100 ng/mL & 94.3 & & 99.1 & & 96.8 & 104 \\
Individual—200 ng/mL & 96.7 & & 101 & & 98.8 & 104 \\
\hline
\end{tabular}

Table 4. Accuracy in matrix.

\begin{tabular}{|c|c|c|c|c|c|c|c|c|}
\hline $100 \mathrm{pg} / \mathrm{mL}$ & & & $200 \mathrm{pg} / \mathrm{mL}$ & & & $300 \mathrm{pg} / \mathrm{mL}$ & & \\
\hline $\mathrm{n}=9$ & AVG (\%) & CV (\%) & $\mathrm{n}=9$ & AVG (\%) & CV (\%) & $n=9$ & AVG (\%) & CV (\%) \\
\hline NDMA & 85.4 & 2.56 & NDMA & 94.0 & 4.68 & NDMA & 94.3 & 4.67 \\
\hline NMEA & 95.5 & 3.94 & NMEA & 104 & 5.18 & NMEA & 102 & 5.32 \\
\hline NDEA & 90.0 & 3.29 & NDEA & 97.4 & 5.16 & NDEA & 97.3 & 4.63 \\
\hline NPIP & 86.6 & 4.37 & NPIP & 92.8 & 4.88 & NPIP & 91.9 & 4.35 \\
\hline NPYR & 111 & 6.80 & NPYR & 106 & 5.03 & NPYR & 100 & 4.55 \\
\hline NMOR & 89.8 & 3.41 & NMOR & 95.6 & 4.43 & NMOR & 95.7 & 4.64 \\
\hline
\end{tabular}


method allows for one of two data analysis processes to occur. Currently, all sample data is analyzed using MassHunter Quantitative Analysis software. The ability to manually integrate peaks from baseline to baseline makes this method the most accurate for samples at low levels, especially around the LODs. The second data analysis process, Indigo Biosystems ASCENT platform, is a more automated process but is still being optimized. The peak fitting and peak picking algorithms utilized on this platform work well for higher concentration samples. The one main impediment to using this platform thus far is the inability to adjust peak baselines; the software only allows for two data points to be chosen on the chromatogram, and the area between is automatically integrated. This has been of greatest concern in samples around the LODs of all analytes: the algorithm for determining the chromatogram baseline creates an undulating baseline, which when picking a small peak can artificially increase or decrease the calculated concentration. Indigo is currently addressing this problem for this method, and if it can be resolved, then the Indigo ASCENT platform will be implemented in the main data analysis process.

\section{Conclusion}

Overall, the newly automated process is time efficient and precise. At least two batches of 48 samples can be prepared each day when higher sample throughput is needed. Automation has improved the overall accuracy and precision. The new sample data flow has improved sample tracking and data analysis, including sample and run quality control evaluation. The sample data flow also enables multiple team members to participate and track sample analysis progress. The entire sample data flow from sample receiving to final result reporting is more efficient while minimizing human errors. This method will be implemented to monitor volatile nitrosamines in population studies such as NHANES.

\section{Acknowledgement}

This study was funded through an interagency agreement by the U.S. Food and Drug Administration Center for Tobacco Products.

\section{References}

[1] Akyuz, M. and Ata, A. (2013) Seasonal Variations of Particle-Associated Nitrosamines by Gas Chromatography-Mass Spectrometry in the Atmospheric Environment of Zonguldak, Turkey. Environmental Science and Pollution Research, 20, 7398-7412. http://dx.doi.org/10.1007/s11356-013-1758-y

[2] Huang, M.C., et al. (2013) Determination of Volatile N-Nitrosamines in Meat Products by Microwave-Assisted Extraction Coupled with Dispersive Micro Solid-Phase Extraction and Gas Chromatography-Chemical Ionisation Mass Spectrometry. Food Chemistry, 138, 227-233.

http://dx.doi.org/10.1016/j.foodchem.2012.09.119

[3] Levallois, P., Ayotte, P., Van Maanen, J.M.S., et al. (2000) Excretion of Volatile Nitrosamines in a Rural Population in Relation to Food and Drinking Water Consumption. Food and Chemical Toxicology, 38, 1013-1019. http://dx.doi.org/10.1016/S0278-6915(00)00089-2

[4] Chowdhury, S. (2014) N-Nitrosodimethylamine (NDMA) in Food and Beverages: A Comparison in Context to Drinking Water. Human and Ecological Risk Assessment, 20, 1291-1312. http://dx.doi.org/10.1080/10807039.2013.817144

[5] Chung, M.J., Lee, S.H. and Sung, N.J. (2002) Inhibitory Effect of Whole Strawberries, Garlic Juice or Kale Juice on Endogenous Formation of N-Nitrosodimethylamine in Humans. Cancer Lett, 182, 1-10. http://dx.doi.org/10.1016/S0304-3835(02)00076-9

[6] Kocak, D., et al. (2012) Determination of Volatile Nitrosamines in Grilled Lamb and Vegetables Using Comprehensive Gas Chromatography_Nitrogen Chemiluminescence Detection. Food Chemistry, 135, 2215-2220. http://dx.doi.org/10.1016/j.foodchem.2012.07.002

[7] Lijinsky, W. (1999) N-Nitroso Compounds in the Diet. Mutation Research/Genetic Toxicology and Environmental Mutagenesis, 443, 129-138. http://dx.doi.org/10.1016/S1383-5742(99)00015-0

[8] Campillo, N., Viñas, P., Martínez-Castillo, N., et al. (2011) Determination of Volatile Nitrosamines in Meat Products by Microwave-Assisted Extraction and Dispersive Liquid-Liquid Microextraction Coupled to Gas ChromatographyMass Spectrometry. Journal of Chromatography A, 1218, 1815-1821. http://dx.doi.org/10.1016/j.chroma.2011.02.010

[9] Ma, F.J., et al. (2012) Occurrence and Source of Nitrosamines and Secondary Amines in Groundwater and its Adjacent Jialu River Basin, China. Environmental Science \& Technology, 46, 3236-3243. http://dx.doi.org/10.1021/es204520b 
[10] Yang, C.S., et al. (1990) Cytochrome P450IIE1: Roles in Nitrosamine Metabolism and Mechanisms of Regulation. Drug Metabolism Reviews, 22, 147-159. http://dx.doi.org/10.3109/03602539009041082

[11] Abdel-Hamid, N.M., Nazmy, M.H. and Abdel-Bakey, A.I. (2011) Polyol Profile as an Early Diagnostic and Prognostic Marker in Natural Product Chemoprevention of Hepatocellular Carcinoma in Diabetic Rats. Diabetes Research and Clinical Practice, 92, 228-237. http://dx.doi.org/10.1016/j.diabres.2011.02.003

[12] Abdel-Hamid, N.M., Nazmy, M.H., Abdel-Ghany, M.I. and Nazmy, W.H. (2012) Cytokines as Important Playmakers of Experimental Hepatocarcinogenesis Confounded by Diabetes. Annals of Hepatology, 11, 118-127.

[13] Arinc, E., Arslan, S., Bozcaarmutlu, A. and Adali, O. (2007) Effects of Diabetes on Rabbit Kidney and Lung CYP2E1 and CYP2B4 Expression and Drug Metabolism and Potentiation of Carcinogenic Activity of $\mathrm{N}$-Nitrosodimethylamine in Kidney and Lung. Food and Chemical Toxicology, 45, 107-118. http://dx.doi.org/10.1016/j.fct.2006.07.026

[14] de la Monte, S.M., Neusner, A., Chu, J. and Lawton, M. (2009) Epidemiological Trends Strongly Suggest Exposures as Etiologic Agents in the Pathogenesis of Sporadic Alzheimer's Disease, Diabetes Mellitus, and Non-Alcoholic Steatohepatitis. Journal of Alzheimer's Disease, 17, 519-529.

[15] de la Monte, S.M. and Tong, M. (2009) Mechanisms of Nitrosamine-Mediated Neurodegeneration: Potential Relevance to Sporadic Alzheimer's Disease. Journal of Alzheimer's Disease, 17, 817-825.

[16] de la Monte, S.M., Tong, M., Lawton, M. and Longato, L. (2009) Nitrosamine Exposure Exacerbates High Fat Diet-Mediated Type 2 Diabetes Mellitus, Non-Alcoholic Steatohepatitis, and Neurodegeneration with Cognitive Impairment. Molecular Neurodegeneration, 4, 54. http://dx.doi.org/10.1186/1750-1326-4-54

[17] Tong, M., Longato, L. and de la Monte, S.M. (2010) Early Limited Nitrosamine Exposures Exacerbate High Fat DietMediated Type 2 Diabetes and Neurodegeneration. BMC Endocrine Disorders, 10, 4. http://dx.doi.org/10.1186/1472-6823-10-4

[18] Tong, M., Neusner, A., Longato, L., et al. (2009) Nitrosamine Exposure Causes Insulin Resistance Diseases: Relevance to Type 2 Diabetes Mellitus, Non-Alcoholic Steatohepatitis, and Alzheimer's Disease. Journal of Alzheimer's Disease, 17, 827-844.

[19] van Maanen, J.M., Welle, I.J., Hageman, G., et al. (1996) Nitrate Contamination of Drinking Water: Relationship with HPRT Variant Frequency in Lymphocyte DNA and Urinary Excretion of $\mathrm{N}$-Nitrosamines. Environmental Health Perspectives, 104, 522-528. http://dx.doi.org/10.1289/ehp.96104522

[20] Yoon, S., Nakada, N. and Tanaka, H. (2012) A New Method for Quantifying $N$-Nitrosamines in Wastewater Samples by Gas Chromatography-Triple Quadrupole Mass Spectrometry. Talanta, 97, 256-261. http://dx.doi.org/10.1016/j.talanta.2012.04.027

[21] Seyler, T.H., Kim, J.G., Hodgson, J.A., et al. (2013) Quantitation of Urinary Volatile Nitrosamines from Exposure to Tobacco Smoke. Journal of Analytical Toxicology, 37, 195-202. http://dx.doi.org/10.1093/jat/bkt020

[22] Ozel, M.Z., Gogus, F., Yagci, S., et al. (2010) Determination of Volatile Nitrosamines in Various Meat Products Using Comprehensive Gas Chromatography-Nitrogen Chemiluminescence Detection. Food and Chemical Toxicology, 48, 3268-3273. http://dx.doi.org/10.1016/j.fct.2010.08.036

[23] Wang, X., Gao, Y.H., Xu, X.J., et al. (2011) Derivatization Method for Determination of Nitrosamines by GC-MS. Chromatographia, 73, 321-327. http://dx.doi.org/10.1007/s10337-010-1871-8

[24] Brisson, I.J., Levallois, P., Tremblay, H., et al. (2013) Spatial and Temporal Occurrence of $N$-Nitrosamines in Seven Drinking Water Supply Systems. Environmental Monitoring and Assessment, 185, 7693-7708. http://dx.doi.org/10.1007/s10661-013-3128-0

[25] Charrois, J.W.A., Arend, M.W., Froese, K.L. and Hrudey, S.E. (2004) Detecting N-Nitrosamines in Drinking Water at Nanogram per Liter Levels Using Ammonia Positive Chemical Ionization. Environmental Science \& Technology, 38, 4835-4841. http://dx.doi.org/10.1021/es049846j

[26] Krauss, M., Longrée, P., Dorusch, F., et al. (2009) Occurrence and Removal of $N$-Nitrosamines in Wastewater Treatment Plants. Water Research, 43, 4381-4391. http://dx.doi.org/10.1016/j.watres.2009.06.048

[27] Krauss, M. and Hollender, J. (2008) Analysis of Nitrosamines in Wastewater: Exploring the Trace Level Quantification Capabilities of a Hybrid Linear Ion Trap/Orbitrap Mass Spectrometer. Analytical Chemistry, 80, 834-842. http://dx.doi.org/10.1021/ac701804y

[28] McDonald, J.A., Harden, N.B., Nghiem, L.D. and Khan, S.J. (2012) Analysis of N-Nitrosamines in Water by Isotope Dilution Gas Chromatography-Electron Ionisation Tandem Mass Spectrometry. Talanta, 99, 146-154. http://dx.doi.org/10.1016/j.talanta.2012.05.032

[29] CLSI (2004) Protocols for Determination of Limits of Detection and Limits of Quantitation; Approved Guideline. CLSI, Wayne, PA.

[30] Caudill, S.P., Schleicher, R.L. and Pirkle, J.L. (2008) Multi-Rule Quality Control for the Age-Related Eye Disease Study. Statistics in Medicine, 27, 4094-4106. http://dx.doi.org/10.1002/sim.3222 


\section{Supplement}

\section{S1. Linearity}

The standard curve shows linearity above 0.999 for a large dynamic range, from 0 to $400 \mathrm{ng} / \mathrm{mL}$, with the lowest non-zero standard concentration being $0.1 \mathrm{ng} / \mathrm{mL}$. The linear range of the analytical method extends from the LOD to the highest calibrator. A weighting factor of $1 / x$ was used for all analytes. A full calibration curve is run with each analytical batch. The calibration curve is rejected if the R-squared value is less than 0.98 for the full dynamic range or the back calculated concentration of any standard is greater than $\pm 10 \%$ of the nominal concentration.

\section{S2. Matrix Equivalency}

To verify matrix equivalency, 10 standard solutions were prepared in ACN (non-matrix). Another 10 standard solutions were prepared in urine (matrix), and carried through sample preparation as described in sample preparation section above. Each calibration curve was run in triplicate, and the average slopes for the two matrices were compared for each analyte. The differences in slope between the matrices for all analytes were below $5 \%$, with NPYR having less than $0.5 \%$ difference (Table S1).

\section{S3. Ruggedness}

To test the ruggedness of both the sample preparation and the GC-QQQ methods, QC samples were prepared and run under different conditions in five parameters, tested separately. These parameters include the number of programmed loops in the pipette mixing step (where one loop is approximately 4 minutes), the volume of DCM added to each well, the volume of prepared sample injected onto the GC, the source temperature of the mass spectrometer, and the percentage of chemical ionization gas (ammonia) in the MS source. Samples were tested for all parameters at below, above, and normal operating conditions. For all parameters tested, less than a 20\% difference in concentration was calculated for all analytes, with the majority well below 10\% (Table S2).

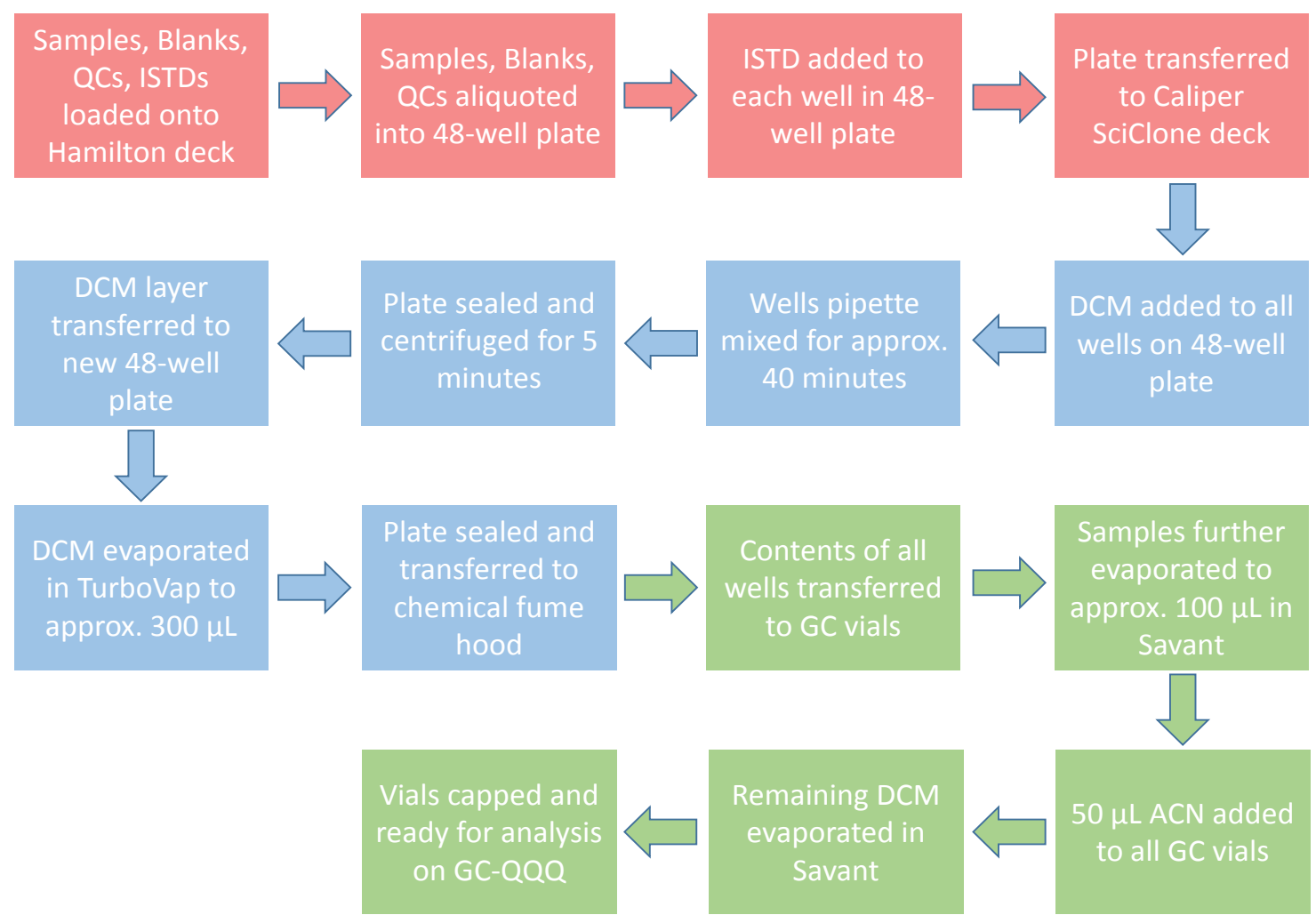

Figure S1. Sample preparation work flow. 
Table S1. Matrix equivalency (All $\mathrm{r}^{2}$ values $>0.999$ ).

\begin{tabular}{cccc}
\hline & Acetonitrile & Urine & \% Diff \\
\cline { 2 - 4 } NDMA & Slope $(\mathrm{n}=3)$ & Slope $(\mathrm{n}=3)$ & 2.79 \\
NMEA & 0.903 & 0.928 & 4.04 \\
NDEA & 2.11 & 2.20 & 3.00 \\
NPIP & 1.14 & 1.17 & 4.79 \\
NPYR & 0.890 & 0.848 & 0.478 \\
NMOR & 1.29 & 1.29 & 2.89 \\
\hline
\end{tabular}

Table S2. Ruggedness.

\begin{tabular}{ccccccc}
\hline & NDMA & NMEA & NDEA & NPIP & NPYR & NMOR \\
\hline Pipette Mixing-8 loops & $58.3(+1.6 \%)$ & $46.5(+5.0 \%)$ & $44.2(+0.9 \%)$ & $40.8(+0.5 \%)$ & $64.5(+4.9 \%)$ & $52.9(+2.7 \%)$ \\
Pipette Mixing-10 loops & 57.4 & 44.3 & 43.8 & 40.6 & 61.5 & 51.5 \\
Pipette Mixing-12 loops & $58.9(+2.6 \%)$ & $46.8(+5.6 \%)$ & $47.0(+7.3 \%)$ & $43.6(+7.4 \%)$ & $61.9(+0.7 \%)$ & $52.7(+2.3 \%)$ \\
DCM volume-2 mL & $60.1(-1.2 \%)$ & $46.0(-2.1 \%)$ & $46.5(-0.6 \%)$ & $41.4(-4.6 \%)$ & $62.9(-0.8 \%)$ & $57.9(+5.5 \%)$ \\
DCM volume-2.5 $\mathrm{mL}^{*}$ & 60.8 & 47.0 & 46.8 & 43.4 & 63.4 & 54.9 \\
DCM volume-3 mL & $59.8(-1.6 \%)$ & $38.4(-18.3 \%)$ & $42.0(-10.3 \%)$ & $35.7(-17.7 \%)$ & $56.3(-11.2 \%)$ & $44.0(-19.9 \%)$ \\
Injection volume-4 $\mu \mathrm{L}$ & $69.0(-5.2 \%)$ & $46.6(+2.0 \%)$ & $51.3(+1.6 \%)$ & $44.1(+1.4 \%)$ & $60.6(-5.6 \%)$ & $53.8(-2.0 \%)$ \\
Injection volume-5 $\mu \mathrm{L}^{*}$ & 72.8 & 45.7 & 50.5 & 43.5 & 64.2 & 54.9 \\
Injection volume-6 $\mu \mathrm{L}$ & $76.8(+5.5 \%)$ & $46.4(+1.5 \%)$ & $50.8(+0.6 \%)$ & $44.1(+1.4 \%)$ & $63.6(-0.9 \%)$ & $53.1(-3.3 \%)$ \\
Source temperature-225 C & $69.8(-7.9 \%)$ & $46.7(+1.7 \%)$ & $50.2(-2.1 \%)$ & $42.4(-3.9 \%)$ & $62.5(-2.6 \%)$ & $52.2(-1.1 \%)$ \\
Source temperature-250 ${ }^{\circ}$ & 75.8 & 45.9 & 51.3 & 44.1 & 64.2 & 52.8 \\
Source temperature-275 ${ }^{\circ} \mathrm{C}$ & $85.0(+12.1 \%)$ & $47.9(+4.4 \%)$ & $51.9(+1.2 \%)$ & $46.6(+5.7 \%)$ & $63.8(-0.6 \%)$ & $53.8(+1.9 \%)$ \\
CI gas-20\% & $62.5(-3.1 \%)$ & $40.7(+3.3 \%)$ & $43.5(-1.4 \%)$ & $37.5(+1.4 \%)$ & $58.2(+2.3 \%)$ & $48.4(+1.7 \%)$ \\
CI gas-25\% ${ }^{*}$ & 64.5 & 39.4 & 44.1 & 37.0 & 56.9 & 47.6 \\
CI gas-30\% & $74.6(+15.7 \%)$ & $40.7(+3.3 \%)$ & $43.2(-2.0 \%)$ & $35.8(-3.2 \%)$ & $57.5(+1.0 \%)$ & $45.9(-3.6 \%)$ \\
\hline
\end{tabular}

All values in $\mathrm{pg} / \mathrm{mL}$. " Denotes current method setting.

\section{S4. Stability}

To ensure the stability of all analytes, two freshly made QC pools $(50 \mathrm{pg} / \mathrm{mL}, 200 \mathrm{pg} / \mathrm{mL})$ were analyzed daily over a 30 day period, with day zero representing the day the pools were made. No significant decrease in concentration is observed for any analyte at either concentration over the duration of the study (Figures S2-S7).

\section{S5. QC Characterization}

The two QC pools used for this method, $50 \mathrm{pg} / \mathrm{mL}$ and $200 \mathrm{pg} / \mathrm{mL}$, were characterized using 20 replicates for each pool over 20 days. QC results were subsequently used to verify methodological precision for each analytical run according to modified Westgard QC rules [30].

\section{S6. Proficiency Testing}

Proficiency testing was conducted by analyzing a series of 5 "blinded" VNA-spiked urine samples every 6 months. PT testing samples were blind-coded by a statistician or QC officer. If blind-analyzed concentrations fell within $20 \%$ of known values for all analyte, and the overall passing rate is $80 \%$ or greater, then the PT was passed. No sample analysis is permitted until PT is passed. 


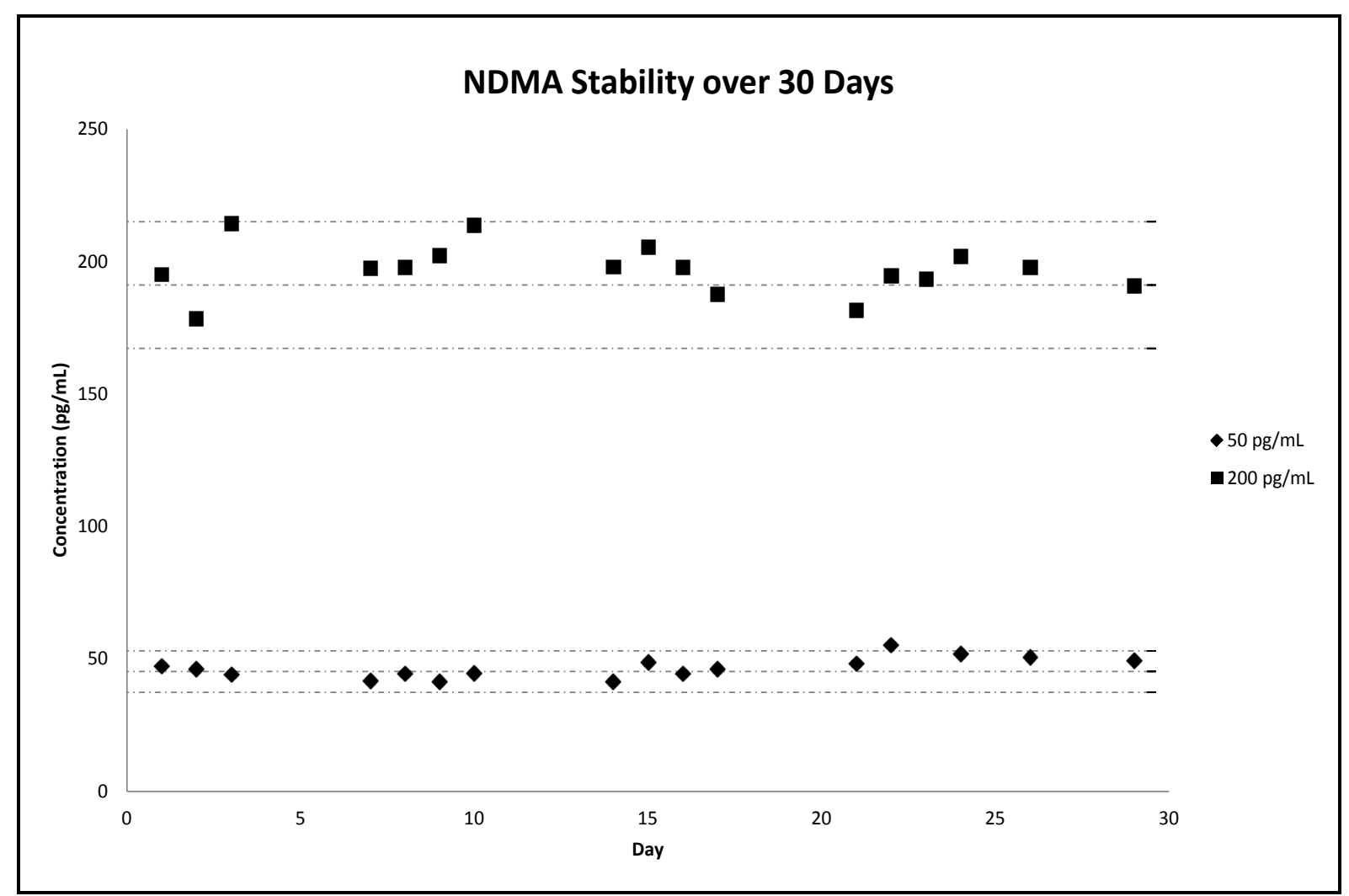

Figure S2. Short-term stability of NDMA.

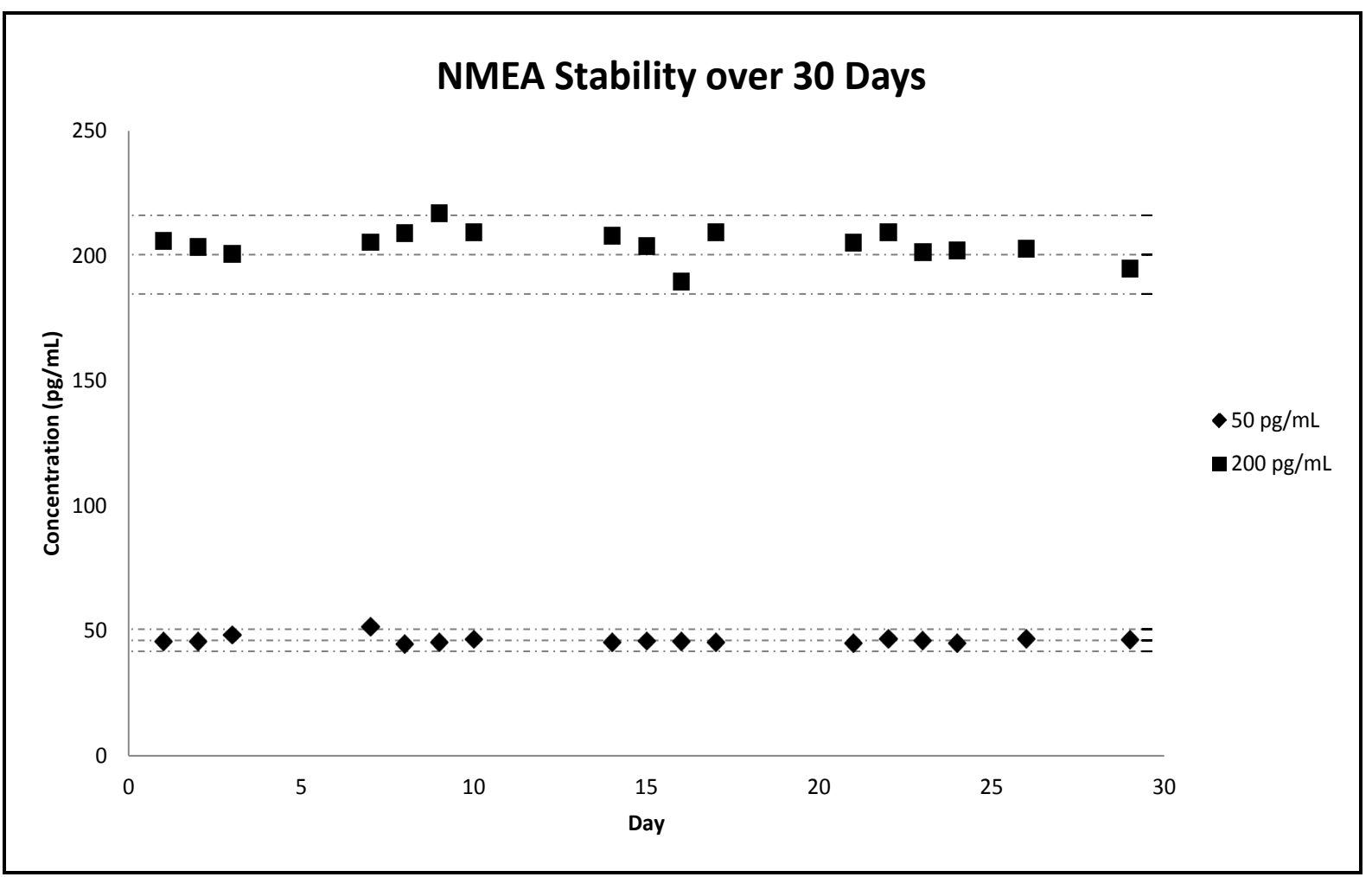

Figure S3. Short-term stability of NMEA. 


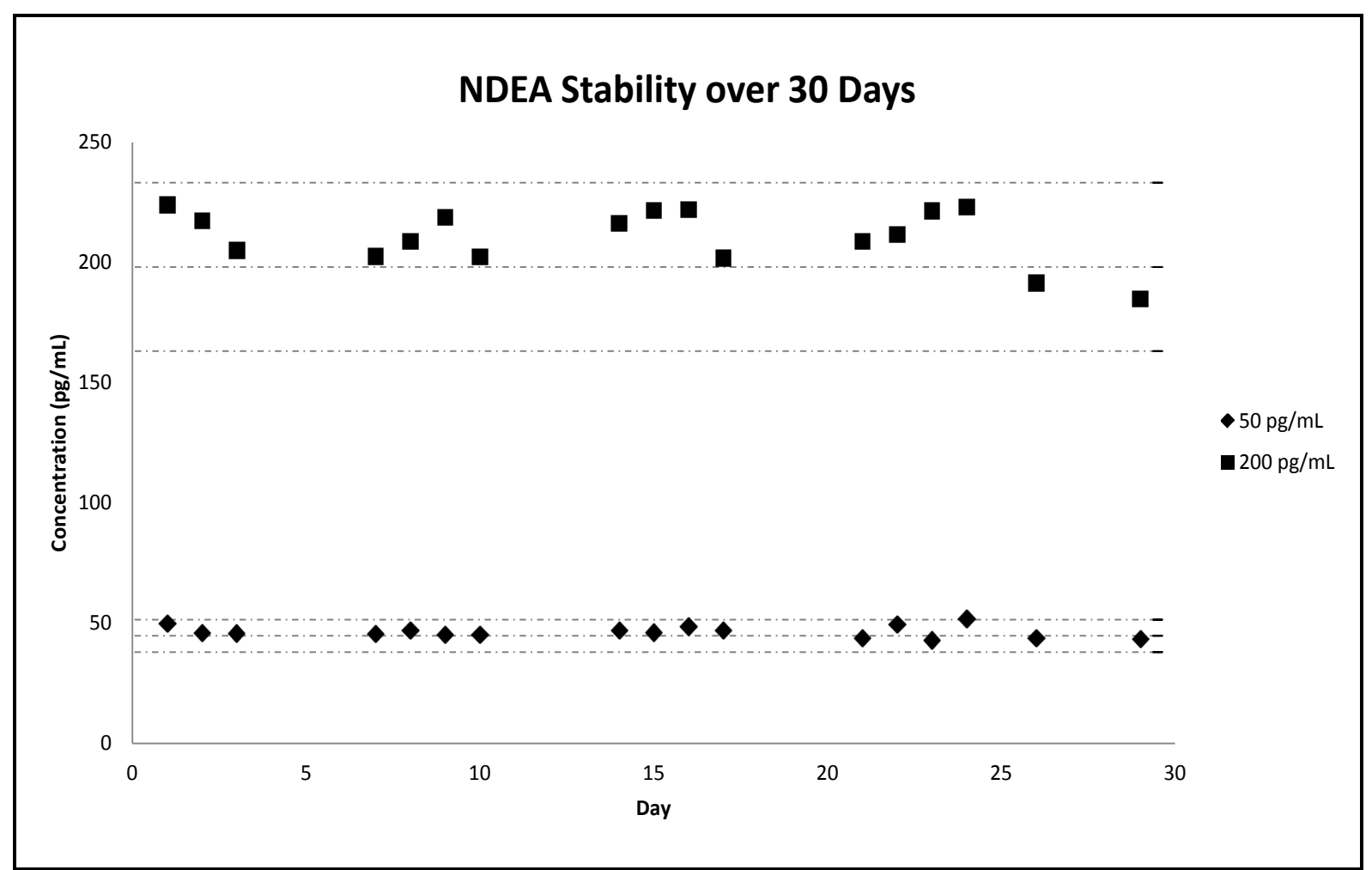

Figure S4. Short-term stability of NDEA.

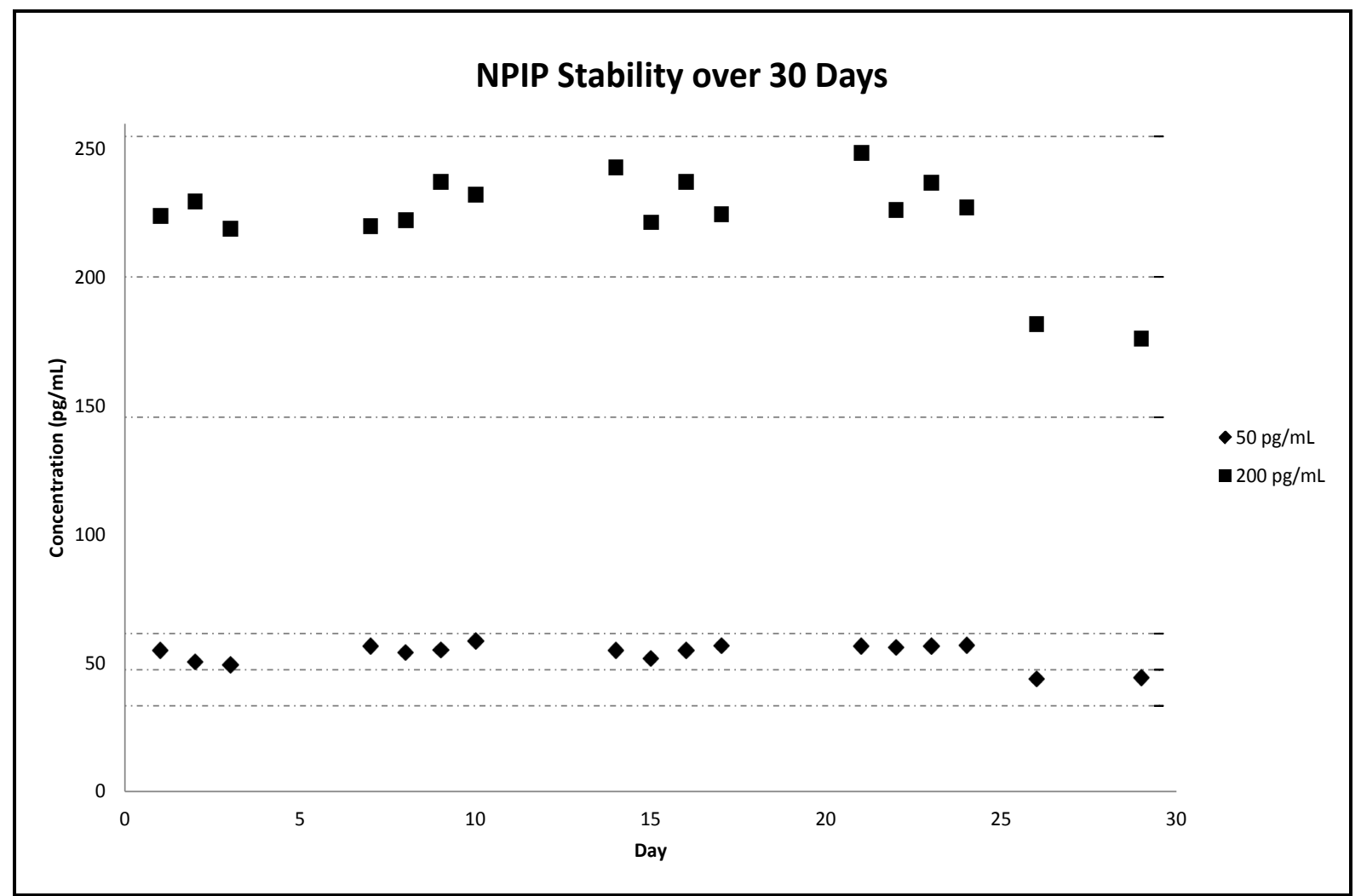

Figure S5. Short-term stability of NPIP. 


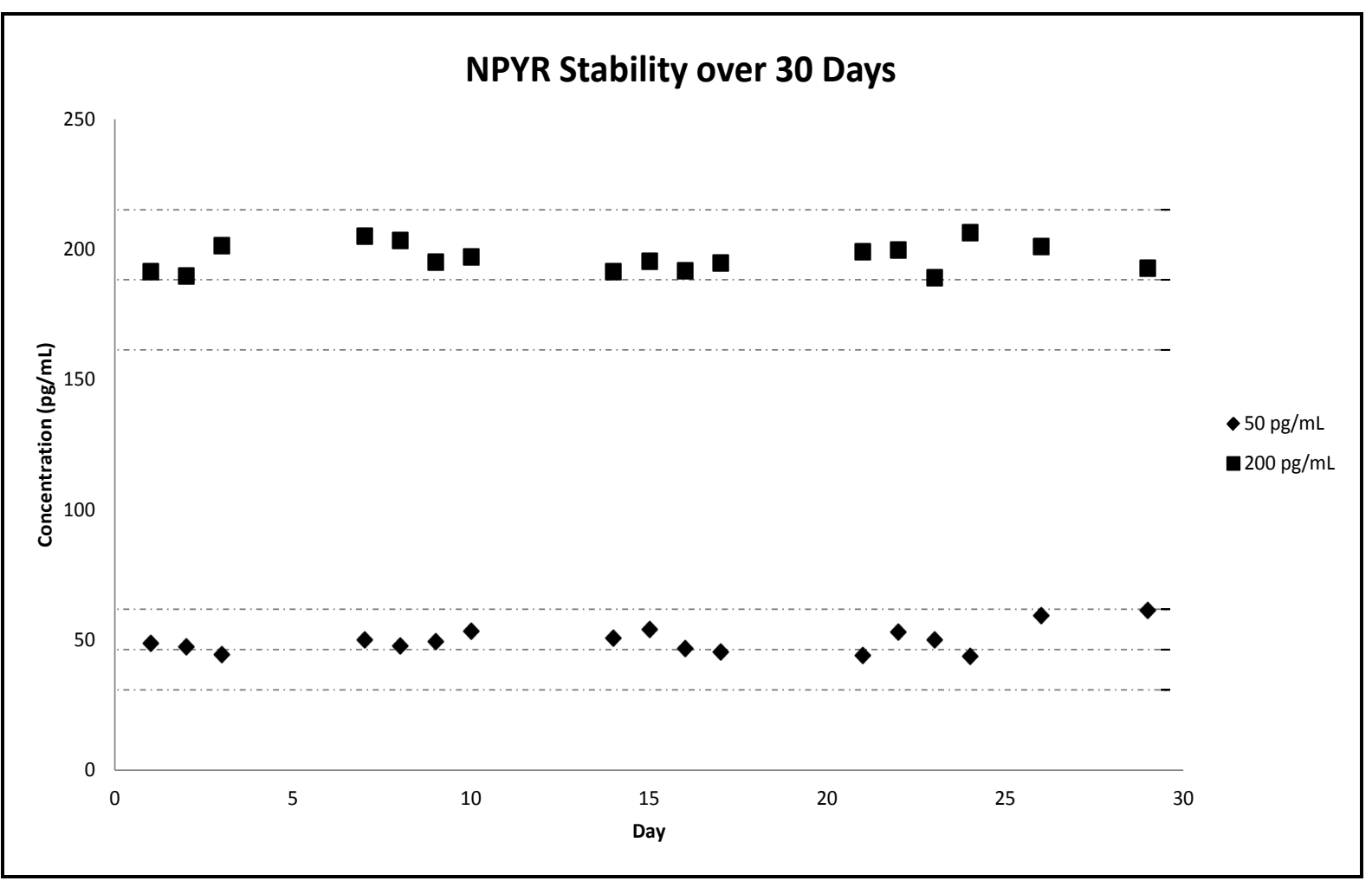

Figure S6. Short-term stability of NPYR.

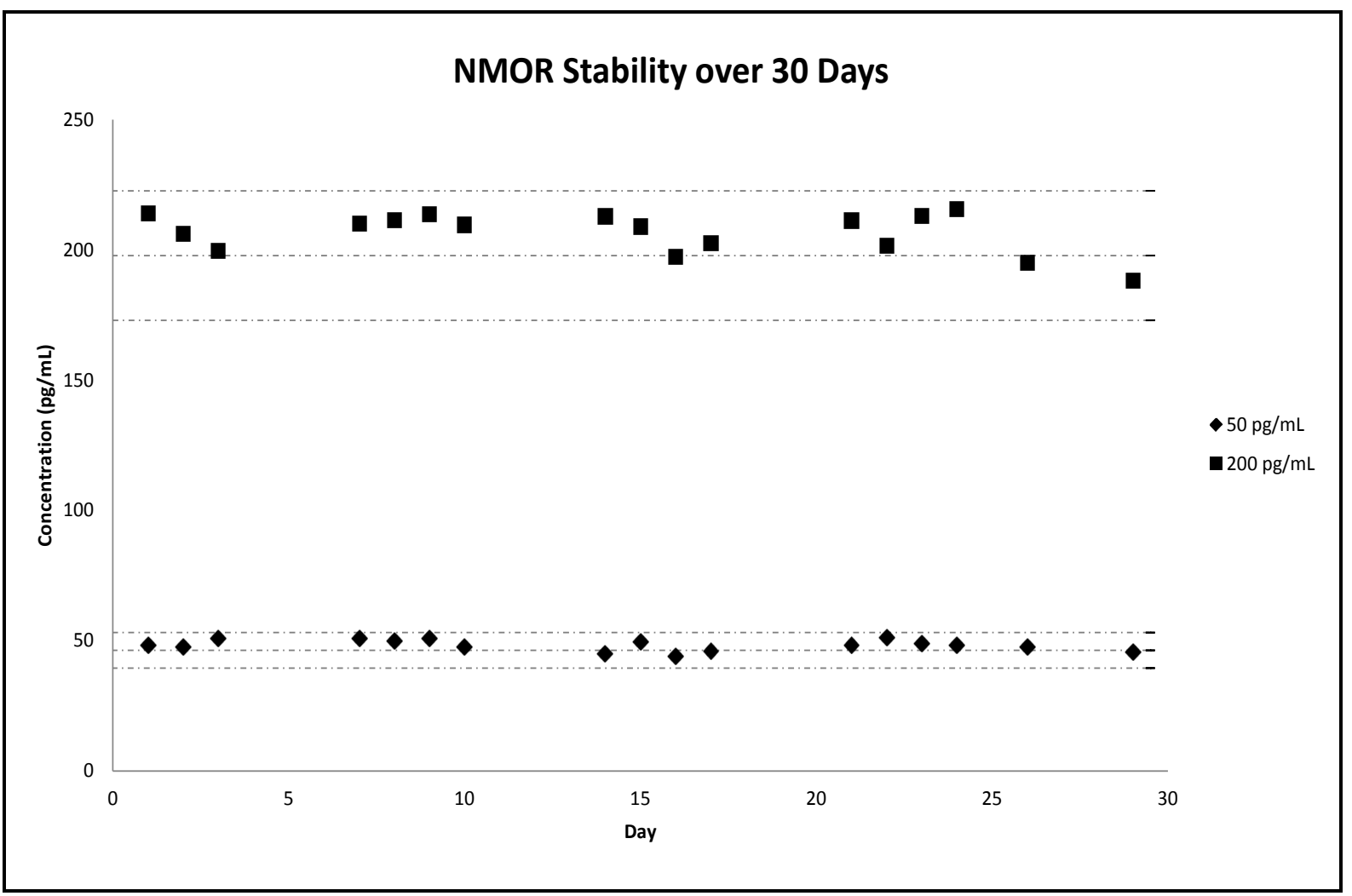

Figure S7. Short-term stability of NMOR. 